\title{
All-arthroscopic reconstruction of the anterior talofibular ligament is comparable to open reconstruction: a systematic review
}

\author{
Ulrike Wittig ${ }^{1}$, Gloria Hohenberger ${ }^{2}$, Martin Ornig ${ }^{1}$, Reinhard Schuh ${ }^{3}$, \\ Andreas Leithner ${ }^{1}$ and Patrick Holweg ${ }^{1}$ \\ ${ }^{1}$ Department of Orthopaedics and Trauma, Medical University of Graz, Graz, Austria \\ 2Department of Trauma, LKH Feldbach-Fürstenfeld, Feldbach, Austria \\ ${ }^{3}$ Department of Orthopaedics, Protestant Hospital Vienna, Vienna, Austria
}

\author{
Correspondence \\ should be addressed \\ to U Wittig \\ Email \\ ulrike.wittig@medunigraz.at
}

- The aim of this study was to determine whether all-arthroscopic repair would lead to improved clinical outcomes, lower complication rates, shorter postoperative immobilization and earlier return to activity compared to open Broström repair in the surgical treatment of chronic lateral ankle instability (CLAl).

- A systematic literature search was conducted using Pubmed and Embase to identify studies dealing with a comparison of outcomes between all-arthroscopic and open Broström repair for CLAI. The search algorithm was 'ankle instability' AND 'Brostrom' AND 'arthroscopic' AND 'open'. The study had to be written in English language, include a direct comparison of all-arthroscopic and open Broström repair to treat CLAI and have full text available.

Exclusion criteria were former systematic reviews, biomechanical studies and case reports.

- Overall, eight studies met the inclusion criteria and were included in the analysis. Clinical outcomes did not differ substantially between patients treated with either arthroscopic or open Broström repair. Studies that reported on return to activity and sports following surgery suggested that patients that had all-arthroscopic Broström repair returned at a quicker rate. Overall complication rate tended to be lower after arthroscopic Broström repair.

- Similar to open repair, all-arthroscopic ligament repair for CLAI is a safe treatment option that yields excellent clinical outcomes.

- Level of Evidence: Level III evidence (systematic review of level I, II and III studies).

\section{Keywords}

- chronic lateral ankle instability

- Broström

- ankle arthroscopy

\section{Introduction}

Lateral ankle sprains are among the most common injuries in sports $(1,2,3)$. The anterior talofibular ligament (ATFL) is the most frequently injured ligament, followed by the calcaneofibular ligament $(\mathrm{CFL})(4,5,6)$. Only in rare cases and following severe traumata, the entire lateral ligament complex ruptures $(7,8)$. In most cases of lateral ligament injury, conservative treatment yields good results, and approximately $80-90 \%$ of patients regain ankle stability by immobilization in a brace $(9,10,11)$. However, about 10 to $20 \%$ develop chronic lateral ankle instability (CLAI) and consequently require surgical ligament repair (12, 13). The Broström technique as well as its modifications is considered the gold standard for treating CLAl. The Broström repair includes a direct suture of the torn ends of the ATFL and CFL $(14,15,16)$. The well-known
Broström-Gould modification by Gould et al. makes use of the inferior extensor retinaculum to support the ATFL remnants and provide a more stable repair (13). Another modification introduced by Karlsson et al. in 1988 suggested reattaching the ATFL and CFL through drill holes, as they were often not completely ruptured, but elongated and scarred (17).

In recent years, interest in less invasive methods to perform lateral ligament repair has increased, including arthroscopic techniques $(18,19,20)$. Arthroscopic-assisted repair combines arthroscopic repair with a percutaneous or mini-open approach $(19,21,22,23,24)$. All-arthroscopic repair involves an anatomical repair of the lateral ligaments in a fully arthroscopic procedure $(25,26,27,28)$. Both methods present the advantage of a minimally invasive 
approach and the chance to address other injury-related pathologies such as synovitis, intra- and periarticular soft-tissue adhesions and removal of loose joint bodies (29). However, arthroscopy is associated with a high complication rate, as sensitive nerve and vessel structures pass within a short distance of the common arthroscopic portals $(30,31)$. Anatomical and clinical studies have shown that the intermediate dorsal cutaneous branch of the superficial peroneal nerve is the most frequently injured nerve during ankle arthroscopy $(32,33,34)$.

Up until now, there is still no evidence whether open or all-arthroscopic lateral ligament repair should be considered as the gold standard.

The aim of this study was to determine whether allarthroscopic repair would lead to improved clinical outcomes, lower complication rates, shorter postoperative immobilization and earlier return to activity compared to open Broström repair in the surgical treatment of CLAI. The authors hypothesized that all-arthroscopic repair would result in improved outcome and earlier return to activity due to the less invasive approach.

\section{Methods}

A systematic literature search according to the Preferred Reporting Items for Systematic Review and Meta-Analysis (PRISMA) guidelines was conducted using Pubmed and
Embase to identify studies dealing with a comparison of outcomes between all-arthroscopic and open Broström repair for CLAI (35). The final search date was February 20,2021 . Search was not restricted by language or year of publication. The search algorithm was 'ankle instability' AND 'Brostrom' AND 'arthroscopic' AND 'open'. Articles with levels I, II, III or IV evidence were regarded eligible. As especially all-arthroscopic lateral ligament repair is still a relatively novel approach and literature on this topic, especially direct comparison to open Broström repair, is still rare, non-randomized cohort studies as well as randomized trials were included. To quantify the risk of selection bias and potential confounders associated with non-randomized trials, a methodological index called MINORS was calculated for all those trials and summarized in Table 1, with a global ideal score of 24 for comparative studies (36). Articles in print journals as well as electronically published studies and conference records were considered eligible for analysis. Manual crosschecking of all references in the accepted papers and other recent reviews was performed in order to supplement the electronic searches and to identify any additional records in the scope of the study topic. Disagreement concerning study choice was resolved by discussion between the first and senior author until agreement was achieved. To satisfy inclusion criteria, the study had to (i) include a direct comparison of all-arthroscopic and open Broström repair to treat CLAl. Moreover, paper (ii) had to be written

Table 1 Overview of included studies.

\begin{tabular}{|c|c|c|c|c|c|c|c|}
\hline Reference & Study design & Level of evidence & Minors, $n$ & Patients, $n$ & Age (years)* & $\begin{array}{c}\text { Gender ratio } \\
(\mathrm{M} / \mathrm{F})\end{array}$ & Follow-up (months)* \\
\hline Zhou et al. (37) & RCS & III & 18 & & & & \\
\hline $\mathrm{Al}$ & & & & 31 & $33.4 \pm 6.4$ & $20 / 11$ & $29.7 \pm 3.4$ \\
\hline $\mathrm{O}$ & & & & 36 & $31.4 \pm 7.8$ & $23 / 13$ & $33.1 \pm 6.8$ \\
\hline Woo et al. (38) & RCS & III & 19 & & & & \\
\hline $\mathrm{Al}$ & & & & 26 & $33.4 \pm 10.6$ & $16 / 10$ & 12 \\
\hline $\mathrm{O}$ & & & & 26 & $31.5 \pm 10.3$ & $16 / 10$ & 12 \\
\hline Zeng et al. (39) & RCS & III & 18 & & & & NA \\
\hline $\mathrm{Al}$ & & & & 17 & $30.9 \pm 6.0$ & $15 / 2$ & \\
\hline $\mathrm{O}$ & & & & 10 & $27.7 \pm 9.7$ & $7 / 3$ & \\
\hline Xu et al. (40) & RCS & III & 22 & & & & \\
\hline $\mathrm{Al}$ & & & & 32 & $33.7 \pm 7.0$ & $24 / 8$ & $36.5 \pm 12.7$ \\
\hline $\mathrm{O}$ & & & & 35 & $35.8 \pm 8.5$ & $25 / 10$ & $39.1 \pm 9.2$ \\
\hline Rigby et al. (41) & RCS & III & 18 & & & & \\
\hline $\mathrm{Al}$ & & & & 30 & $47.9(14-83)$ & $9 / 21$ & $15.6(8.4-20.4)$ \\
\hline $\mathrm{O}$ & & & & 32 & $37.7(9-72)$ & $14 / 18$ & $44.4(15.6-63.6)$ \\
\hline Li et al. (42) & RCS & III & 20 & & & & \\
\hline $\mathrm{Al}$ & & & & 23 & $30.3 \pm 10.1$ & $18 / 5$ & $39.7 \pm 10.3$ \\
\hline $\mathrm{O}$ & & & & 37 & $28.7 \pm 8.7$ & $29 / 8$ & $35.5 \pm 9.9$ \\
\hline Yeo et al. (43) & $\mathrm{RCT}$ & I & & & & & \\
\hline $\mathrm{Al}$ & & & & 25 & $35.2(19-54)$ & $7 / 18$ & 12 \\
\hline $\mathrm{O}$ & & & & 23 & $34.3(17-52)$ & $12 / 11$ & 12 \\
\hline Matsui et al. (44) & $\mathrm{RCS}$ & III & 17 & & & & \\
\hline $\mathrm{Al}$ & & & & 19 & $28(8-59)$ & $12 / 7$ & 12 \\
\hline $\mathrm{O}$ & & & & 18 & $24(13-56)$ & $8 / 10$ & 12 \\
\hline
\end{tabular}

*Data are presented as mean \pm S.D. or as mean (range).

AA, arthroscopically assisted Broström; Al, all-inside arthroscopic Broström; F, female; M, male; NA, not applicable; O, open Broström; RCS, retrospective cohort study; RCT, randomized controlled trial. 
in English language and (iii) full text had to be provided. Exclusion criteria were (i) former systematic reviews, (ii) cadaveric or biomechanical studies and (iii) case reports.

Literature search and consequently study selection were performed by the first author of the study. Articles considered eligible were then reviewed by both the first and senior authors.

\section{Statistical analysis}

Statistical analysis was performed using Review Manager Version 5.4 software (The Cochrane Collaboration). To quantify heterogeneity between studies, the $\mathrm{I}^{2}$ statistic was conducted and forest plots were used for graphical representation (22). Low heterogeneity was indicated by an $\mathrm{I}^{2}$ value of $<25 \%$, while high heterogeneity was determined by an $\mathrm{I}^{2}$ value of $>75 \%$. If the $\mathrm{I}^{2}$ value was $>50 \%$, a random-effects model was used; otherwise, a fixed-effects model was applied. A descriptive summary of demographic data of the respective patient cohorts was depicted as frequency (absolute numbers, percentage) for qualitative variables and mean, S.D. and range for quantitative variables.

\section{Results}

The electronic database search using the above-mentioned keywords revealed 93 studies. After the removal of duplicates, 53 articles were left to be considered. Through manual cross-referencing, two additional records were identified. After applying the predefined inclusion and exclusion criteria and screening by title and abstract, 36 articles were left potentially eligible. After review of the full-texts, 28 studies were excluded, as they were not in the scope of this review, leaving 8 papers eligible. Finally, eight studies were included in the analysis. The PRISMA flow diagram of the search algorithm is depicted in Fig. 1.

Full text articles of the included studies were either downloaded from the respective journal's website or inquired via the author's own online library.

Of the included studies, seven were retrospective cohort studies and one was a randomized controlled trial. A summary of the study characteristics is depicted in Table 1.

Table 2 summarizes the clinical outcomes measured by different scores including American Orthopaedic Foot and Ankle Society Ankle-Hindfoot Score (AOFAS), visual analog scale (VAS) and the Karlsson Score.

\section{Clinical outcome scores}

Postoperative AOFAS scores were reported in seven studies (Zhou et al. (37), Woo et al. (38), Zeng et al. (39), Xu et al. (40), Rigby et al. (41), Li et al. (42), Yeo et al.

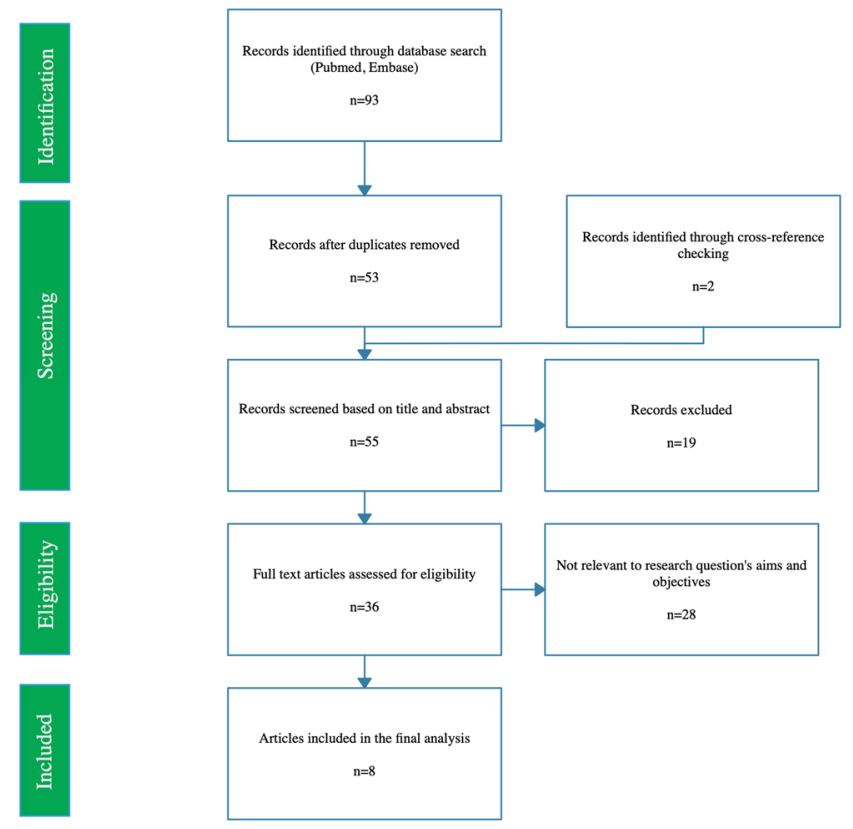

Figure 1

PRISMA flow diagram of the identification of relevant studies.

(43)). VAS scores were reported in six studies (Zhou et al. (37), Woo et al. (38), Xu et al. (40), Rigby et al. (41), Yeo et al. (43), Matsui et al. (44)). The forest plots are depicted in Figs 2 and 3 . The Karlsson score was presented in six studies (Zhou et al. (37), Zeng et al. (39), Xu et al.

Table 2 Evaluation of clinical outcomes. Data are presented mean \pm S.D., mean (S.E.M.) or as mean (range).

\begin{tabular}{|c|c|c|c|}
\hline Reference & AOFAS & VAS & Karlsson score \\
\hline \multicolumn{4}{|l|}{ Zhou et al. (37) } \\
\hline $\mathrm{Al}$ & $91.71 \pm 5.46$ & $1.74 \pm 1.24$ & $87.52 \pm 7.59$ \\
\hline $\mathrm{O}$ & $90.67 \pm 5.59$ & $1.58 \pm 1.2$ & $88.75 \pm 5.56$ \\
\hline \multicolumn{4}{|l|}{ Woo et al. (38) } \\
\hline $\mathrm{Al}$ & $94.2 \pm 10.0$ & $1.2 \pm 2.7$ & \\
\hline $\mathrm{O}$ & $70.9 \pm 33.1$ & $2.1 \pm 2.6$ & \\
\hline \multicolumn{4}{|l|}{ Zeng et al. (39) } \\
\hline $\mathrm{Al}$ & $92.4 \pm 5.9$ & & $89.2 \pm 8.4$ \\
\hline $\mathrm{O}$ & $91.1 \pm 6.2$ & & $90.5 \pm 8.8$ \\
\hline \multicolumn{4}{|l|}{ Xu et al. (40) } \\
\hline $\mathrm{Al}$ & $87.7 \pm 7.6$ & $1.8 \pm 1.6$ & $83.1 \pm 8.2$ \\
\hline $\mathrm{O}$ & $86.9 \pm 7.3$ & $2.1 \pm 1.7$ & $81.7 \pm 9.1$ \\
\hline \multicolumn{4}{|l|}{ Rigby et al. (41) } \\
\hline $\mathrm{Al}$ & $95.33(55-100)$ & $1.5(0-10)$ & $91.8(55-100)$ \\
\hline $\mathrm{O}$ & $93.53(49-100)$ & $1.2(0-9.5)$ & $93.41(54-100)$ \\
\hline \multicolumn{4}{|l|}{ Li et al. (42) } \\
\hline $\mathrm{Al}$ & $93.3 \pm 8.9$ & & $90.3 \pm 12.5$ \\
\hline $\mathrm{O}$ & $92.4 \pm 8.6$ & & $89.4 \pm 10.6$ \\
\hline \multicolumn{4}{|l|}{ Yeo et al. (43) } \\
\hline $\mathrm{Al}$ & $90.3(2.4)$ & $1.7(0.4)$ & $76.2(2.8)$ \\
\hline $\mathrm{O}$ & $89.2(2.3)$ & $2.0(0.4)$ & $73.5(2.8)$ \\
\hline Matsui et al. (44) & NA & & \\
\hline $\mathrm{Al}$ & & $1.24(0-5.5)$ & \\
\hline $\mathrm{O}$ & & $1.92(0.4-6.2)$ & \\
\hline
\end{tabular}

Al, all-inside arthroscopic Broström; AA, arthroscopically assisted Broström; O, open Broström; TTA, talar tilt angle. 


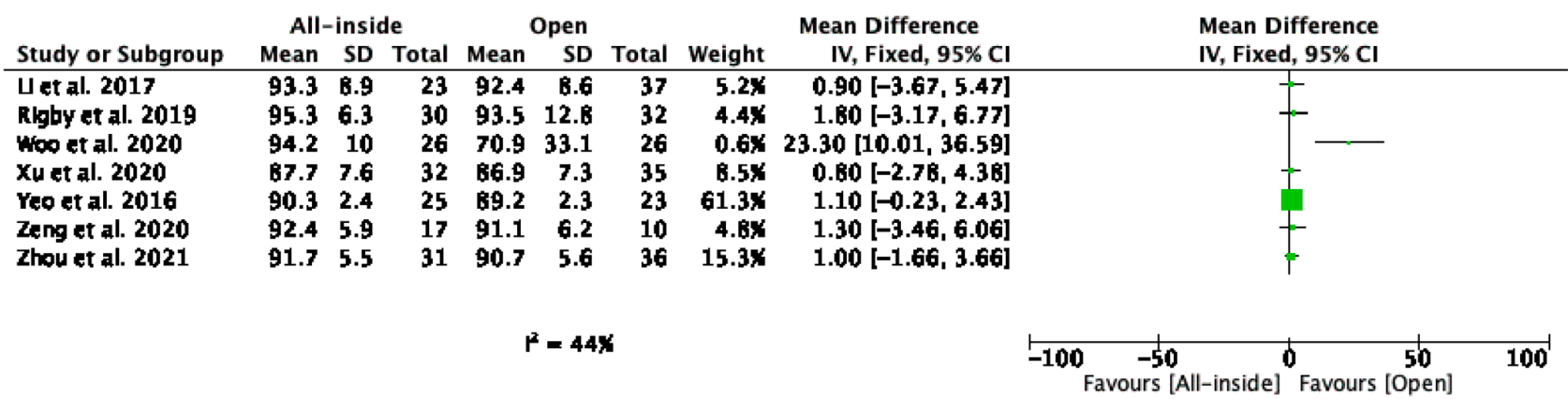

\section{Figure 2}

Results of aggregate analysis for the comparison of AOFAS scores between Al and O groups. IV, inverse variance.

(40), Rigby et al. (41), Li et al. (42), Yeo et al. (43)). The forest plot is depicted in Fig. 4. None of the three reported clinical outcome scores tended to be substantially in favor of either arthroscopic or open repair.

\section{Postoperative treatment and return to activity}

An overview of postoperative treatment protocols and return to sports is given in Table 3. Seven of the eight included studies applied the same postoperative protocols to both patient collectives, whether Broström repair was performed arthroscopically or open.

In the study by Rigby et al. (41), different postoperative rehabilitation protocols were applied. In their study, mean time to weightbearing amounted to 12 days (range, 9-16 days) in the arthroscopic group and was thus substantially shorter than in the open Broström group, where the mean time to weightbearing was 22 days (range, 20-26 days). However, eventually, $97 \%$ were able to return to full activity compared to before the lateral ligament injury happened, which was equal for both groups.

In the study by Matsui et al. (44), mean return to daily activity and mean return to sports were calculated. Return to daily activity was significantly shorter in the arthroscopic Broström group with a mean of 5.3 weeks (range, 3-12 weeks) compared to 7.1 weeks (range, 5-12 weeks) in the open group $(P<0.05)$. Moreover, return to sports was also faster in the arthroscopic Broström group with a mean of 16.5 weeks (range, 12-22 weeks) versus 17.1 weeks (range, 13-22 weeks) in the open group, although not statistically significant $(P=0.07)$.

In most studies, even partial weightbearing was prohibited for the first 2-6 weeks. However, in the study by Matsui et al. (44), weightbearing as tolerated was permitted immediately after surgery in both the arthroscopic and the open Broström group.

\section{Complication rates}

Complication rates, especially concerning wound healing, irritation of the peroneal nerve, knot pain and the need for revision surgery, were reported in all included trials. An overview is given in Table 4. The concomitant forest plots are depicted in Figs 5, 6, 7 and 8. Overall complication rate tended to be lower after arthroscopic Broström repair. Moreover, impaired wound healing tended to occur more often after open Broström repair. On the other hand, irritations of the peroneal nerve and knot pain tended to be more likely after arthroscopic Broström repair.

\section{Discussion}

One of the most important findings of this study was that clinical outcomes did not differ substantially between

\begin{tabular}{|c|c|c|c|c|c|c|c|c|c|}
\hline \multirow[b]{2}{*}{ Study or Subgroup } & \multicolumn{3}{|c|}{ All-inside } & \multicolumn{3}{|c|}{ Open } & \multicolumn{2}{|r|}{ Mean Difference } & \multirow{2}{*}{$\begin{array}{l}\text { Mean Difference } \\
\text { IV, Fixed, } 95 \% \mathrm{CI}\end{array}$} \\
\hline & Mean & SD & Total & Mean & SD & Total & Weight & IV, Fixed, $95 \% \mathrm{CI}$ & \\
\hline Matsul et al. 2016 & 1.2 & 0.9 & 19 & 1.9 & 1 & 1B & $9.7 x$ & $-0.70[-1.31,-0.09]$ & \\
\hline Rkgy et al. 2019 & 1.5 & 1.7 & 30 & 1.2 & 1.6 & 0 & & Not estimable & \\
\hline Woo et al. 2020 & 1.2 & 2.7 & 26 & 2.1 & 2.6 & 26 & 1. $\mathbf{B X}$ & $-0.90[-2.34,0.54]$ & \\
\hline$x u$ et al. 2020 & 1.8 & 1.6 & 32 & 2.1 & 1.7 & 35 & $5.9 \%$ & $-0.30[-1.09,0.49]$ & \\
\hline Yeo et al. 2016 & 1.7 & 0.4 & 25 & 2 & 0.4 & 23 & $71.6 x$ & $-0.30[-0.53,-0.07]$ & \\
\hline Zhou et al. 2021 & 1.7 & 1.2 & 31 & 1.6 & 1.2 & 36 & $11.1 \times$ & $0.10[-0.4 B, 0.6 B]$ & \\
\hline
\end{tabular}

\section{$\mathbf{r}=\mathbf{3 x}$}

$-100 \underset{-50}{-50} \stackrel{0}{50} 100$
Favours [All-inside] Favours [Open]

Figure 3

Results of aggregate analysis for the comparison of total VAS scores between Al and O groups. IV, inverse variance. 


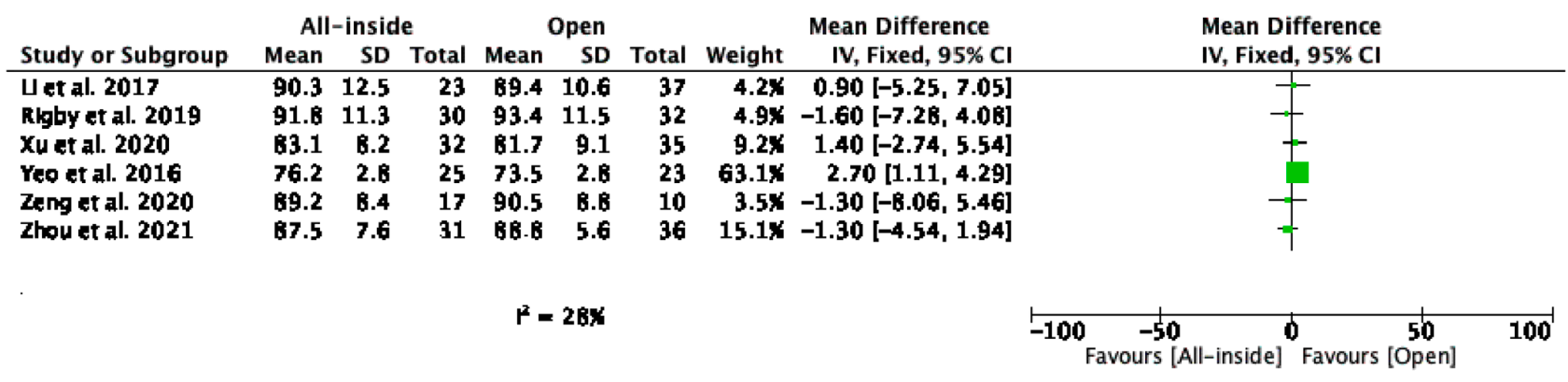

Figure 4

Results of aggregate analysis for the comparison of Karlsson scores between Al and O groups. IV, inverse variance.

patients treated with either arthroscopic or open Broström repair. Although return to activity and sports were not calculated in all studies, they tended to be substantially faster in patients treated with arthroscopic Broström repair. Concerning complications, knot pain and peroneal nerve irritation were common but failed to reach statistical significance. On the other hand, the overall complication rate and impairment of wound healing tended to be lower after all-arthroscopic Broström repair. This implies that allarthroscopic repair can be seen as a safe technique.

Historically, the open Broström-Gould technique has been the preferred technique for lateral ankle ligament repair. First described in 1980, it comprises direct reconstruction of the ATFL and CFL by enhancement with the joint capsule and use of the inferior extensor retinaculum (13). In recent years, minimally invasive

Table 3 Evaluation of postoperative treatment and rehabilitation protocols.

\begin{tabular}{|c|c|}
\hline Reference & Postoperative mobilization \\
\hline \multicolumn{2}{|c|}{ Zhou et al. (37) } \\
\hline \multirow[t]{3}{*}{ Al \& O } & Short cast for 2 weeks \\
\hline & Ankle brace for 4 weeks \\
\hline & $\begin{array}{l}\text { Partial weightbearing } 4 \text { weeks after surgery, followed by progressive } \\
\text { weightbearing }\end{array}$ \\
\hline \multicolumn{2}{|c|}{ Woo et al. (38) } \\
\hline \multirow[t]{2}{*}{ Al \& O } & Posterior splint, non-weightbearing for 2 weeks \\
\hline & Full weightbearing after 2 weeks, walking boot for 4 weeks \\
\hline \multicolumn{2}{|c|}{ Zeng et al. (39) } \\
\hline \multirow[t]{2}{*}{ Al \& O } & Short cast for 2 weeks, followed by walking boot \\
\hline & Full weightbearing after 6-12 weeks \\
\hline \multicolumn{2}{|c|}{ Xu et al. (40) } \\
\hline \multirow[t]{3}{*}{$A I \& O$} & Ankle brace for 6 weeks \\
\hline & Partial weight bearing after 4 weeks \\
\hline & Full weightbearing after 6 weeks \\
\hline \multicolumn{2}{|c|}{ Rigby et al. (41) } \\
\hline \multirow[t]{3}{*}{$\mathrm{Al}$} & Below knee splint for 10 days non-weightbearing \\
\hline & Short leg cast $50 \%$ weightbearing for 10 days \\
\hline & Full weightbearing in a walking cast for $7-10$ days \\
\hline \multirow[t]{2}{*}{$\mathrm{O}$} & Splint for 10-14 days non-weightbearing \\
\hline & Progressive weightbearing in a cast or boot after 3 weeks \\
\hline \multicolumn{2}{|c|}{ Li et al. (42) } \\
\hline \multirow[t]{3}{*}{$\mathrm{Al} \& \mathrm{O}$} & Short leg cast for 2 weeks \\
\hline & Ankle brace after 2 weeks \\
\hline & Weightbearing permitted after 4 weeks \\
\hline \multicolumn{2}{|c|}{ Yeo et al. (43) } \\
\hline \multirow[t]{3}{*}{ Al \& O } & Posterior splint for 2 weeks non-weightbearing \\
\hline & Short-leg walking cast for 2 weeks with progressive weightbearing \\
\hline & Splint for 2 weeks \\
\hline \multicolumn{2}{|c|}{ Matsui et al. (44) } \\
\hline Al \& O & $\begin{array}{l}\text { Splint for several days, immediate weightbearing, followed by ankle brace for } \\
6-8 \text { weeks }\end{array}$ \\
\hline \multicolumn{2}{|l|}{$\mathrm{Al}$} \\
\hline $\mathrm{O}$ & \\
\hline
\end{tabular}

Return to activity*

Full return allowed after 3 months

Mean time (days) to weightbearing: 12 (9-16)

$97 \%$ returned to full activity

Mean time (days) to weightbearing: 22 (20-26)

$97 \%$ returned to full activity

Straight running allowed after 8 weeks

Full return after 12 weeks

Return (weeks) to daily activity: $5.3(3-12)$

Return (weeks) to sports: 16.5 (12-22)

Return (weeks) to daily activity: $7.1(5-12) ;(P<0.05)$

Return (weeks) to sports: $17.1(13-22) ;(P=0.07)$

*Data presented as mean (range). 
Table 4 Complication rates.

\begin{tabular}{|c|c|c|}
\hline Reference & Complication rate, $\%$ & $\begin{array}{l}\text { Impaired wound } \\
\text { healing, } \%\end{array}$ \\
\hline \multicolumn{3}{|c|}{ Zhou et al. (37) } \\
\hline $\mathrm{Al}$ & $6.5(2 / 31)$ & $0.0(0 / 31)$ \\
\hline $\mathrm{O}$ & $11.1(4 / 36)$ & $0.0(0 / 36)$ \\
\hline \multicolumn{3}{|c|}{ Woo et al. (38) } \\
\hline $\mathrm{Al}$ & $0.0(0 / 26)$ & $0.0(0 / 26)$ \\
\hline $\mathrm{O}$ & $0.0(0 / 26)$ & $0.0(0 / 26)$ \\
\hline \multicolumn{3}{|c|}{ Zeng et al. (39) } \\
\hline $\mathrm{Al}$ & $11.8(2 / 17)$ & $5.9(1 / 17)$ \\
\hline $\mathrm{O}$ & $30.0(3 / 10)$ & $20(2 / 10)$ \\
\hline \multicolumn{3}{|c|}{ Xu et al. (40) } \\
\hline $\mathrm{Al}$ & $15.6(5 / 32)$ & $0.0(0 / 32)$ \\
\hline $\mathrm{O}$ & $14.3(5 / 35)$ & $5.7(2 / 35)$ \\
\hline \multicolumn{3}{|c|}{ Rigby et al. (41) } \\
\hline $\mathrm{Al}$ & $6.7(2 / 30)$ & $0.0(0 / 30)$ \\
\hline $\mathrm{O}$ & $6.3(2 / 32)$ & $0.0(0 / 32)$ \\
\hline \multicolumn{3}{|l|}{ Li et al. (42) } \\
\hline $\mathrm{Al}$ & $4.3(1 / 23)$ & $0.0(0 / 23)$ \\
\hline $\mathrm{O}$ & $5.4(2 / 37)$ & $0.0(0 / 37)$ \\
\hline \multicolumn{3}{|c|}{ Yeo et al. (43) } \\
\hline $\mathrm{Al}$ & $20.0(5 / 25)$ & $0.0(0 / 25)$ \\
\hline $\mathrm{O}$ & $13.0(3 / 23)$ & $4.3(1 / 23)$ \\
\hline \multicolumn{3}{|c|}{ Matsui et al. (44) } \\
\hline $\mathrm{Al}$ & $10.5(2 / 19$ & $0.0(0 / 19)$ \\
\hline $\mathrm{O}$ & $22.2(4 / 18)$ & $16.7(3 / 18)$ \\
\hline
\end{tabular}

\begin{tabular}{|c|c|c|}
\hline $\begin{array}{c}\text { Irritation of peroneal } \\
\text { nerve, } \%\end{array}$ & Knot pain, \% & Revision surgery, \% \\
\hline $3.2(1 / 31)$ & $0.0(0 / 31)$ & $0.0(0 / 31)$ \\
\hline $2.8(1 / 36)$ & $2.8(1 / 36)$ & $0.0(0 / 36)$ \\
\hline $0.0(0 / 26$ & $0.0(0 / 26)$ & $0.0(0 / 26)$ \\
\hline $0.0(0 / 26)$ & $0.0(0 / 26)$ & $0.0(0 / 26)$ \\
\hline $5.9(1 / 17)$ & $0.0(0 / 17)$ & $0.0(0 / 17)$ \\
\hline $0.0(0 / 10)$ & $10.0(1 / 10)$ & $0.0(0 / 10)$ \\
\hline $9.4(3 / 32)$ & $6.3(2 / 32)$ & $0.0(0 / 32)$ \\
\hline $5.7(2 / 35)$ & $0.0(0 / 35)$ & $0.0(0 / 35)$ \\
\hline $3.3(1 / 30)$ & $0.0(0 / 30)$ & $0.0(0 / 30)$ \\
\hline $6.3(2 / 32)$ & $0.0(0 / 32)$ & $0.0(0 / 32)$ \\
\hline $0.0(0 / 23)$ & $0.0(0 / 23)$ & $0.0(0 / 23)$ \\
\hline $0.0(0 / 37)$ & $0.0(0 / 37)$ & $0.0(0 / 37)$ \\
\hline $12.0(3 / 25)$ & $8.0(2 / 25)$ & $4.0(1 / 25)$ \\
\hline $8.7(2 / 23)$ & $0.0(0 / 23)$ & $0.0(0 / 23)$ \\
\hline $10.5(2 / 19)$ & $0.0(0 / 19)$ & $0.0(0 / 19)$ \\
\hline $5.6(1 / 18)$ & $0.0(0 / 18)$ & $0.0(0 / 18)$ \\
\hline
\end{tabular}

techniques have become more and more popular in order to achieve enhanced wound healing and thereby also accelerated return to activity. One advantage of arthroscopic ligament repair is that intraarticular pathologies such as synovitis or free joint bodies can be addressed simultaneously $(18,41,45)$. Ferkel et al. reported that $93 \%$ of patients treated arthroscopically for lateral ankle instability showed intraarticular abnormalities. In another study by Slater et al., 29\% of patients presented with chondral injuries, $24 \%$ with loose bodies and $41 \%$ showed anterior osteophytes. A biomechanical study showed that arthroscopic repair using suture anchors was able to achieve comparable results to open repair
(46). There are few studies that report on biomechanical durability and long-term revision rate after arthroscopic repair. Other important factors to consider regarding the all-arthroscopic technique include the risk of injury to the superficial peroneal or sural nerve and also irritation by suture knots or prominent anchors $(23,47)$. Studies have shown modifications to avoid irritation by prominent suture anchors such as knotless anchors or bioabsorbable anchors $(23,25)$. Anatomical studies have tried to define safe zones for ankle arthroscopy to avoid nerve damage $(48,49,50)$. Nevertheless, although peroneal nerve irritations occurred a bit more often after all-arthroscopic repair according to the findings of our study, this slight

\begin{tabular}{|c|c|c|c|c|c|c|}
\hline \multirow[b]{2}{*}{ Study or Subgroup } & \multicolumn{2}{|c|}{ All-inside } & \multicolumn{2}{|c|}{ Open } & \multirow[b]{2}{*}{ Weight } & \multirow{2}{*}{$\begin{array}{c}\text { Odds Ratio } \\
\mathrm{M}=\mathrm{H} \text {, Fixed, } 95 \% \mathrm{CI}\end{array}$} \\
\hline & Events & Total & Events & Total & & \\
\hline U et al. 2017 & 1 & 23 & 2 & 37 & $7.2 x$ & $0.80[0.07,9.3$ \\
\hline Matsul et al. 2016 & 2 & 19 & 4 & 1B & 1B.1\% & $0.41[0$ \\
\hline Rigby et al. 2019 & 2 & 30 & 2 & 32 & $0.9 x$ & $1.07[0.14,8$. \\
\hline Woo et al. 2020 & 0 & 26 & 0 & 26 & & Not \\
\hline Xuet al. 2020 & 5 & 32 & 5 & 35 & 15 & $1.11[0$ \\
\hline 2016 & 5 & 25 & 3 & 23 & $12.3 \%$ & $1.67[0.35,7$. \\
\hline 2020 & 2 & 17 & 3 & 10 & $16.4 \%$ & $0.31[0.04,2$ \\
\hline Zhou et al. 2021 & 2 & 31 & 4 & 36 & $17.1 \times$ & $0.55[0.09,3$ \\
\hline
\end{tabular}

$r^{2}=0 x$

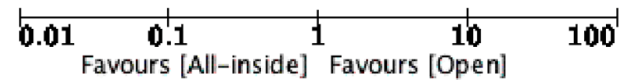

Figure 5

Results of aggregate analysis for the comparison of overall complication rates between BR and ST groups. Numbers for 'events' refer to failure; numbers for 'total' refer to total participants. $\mathrm{M}-\mathrm{H}$, Mantel-Haenszel method. 


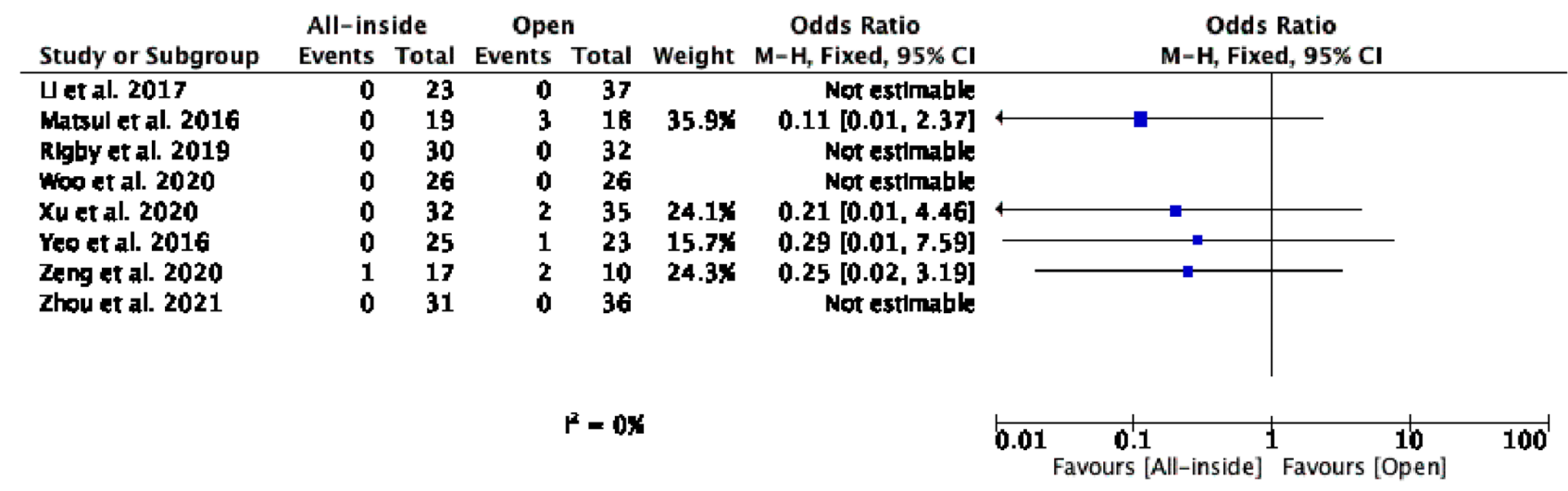

\section{Figure 6}

Results of aggregate analysis for the comparison of rates for irritation of woundhealing between BR and ST groups. Numbers for 'events' refer to failure; numbers for 'total' refer to total participants. M-H, Mantel-Haenszel method.

difference was far from statistical significance, indicating that all-arthroscopic technique is a safe procedure.

Through the database search used in this study, only one comparative study contrasting patients treated with allarthroscopic or arthroscopic-assisted Broström repair has been published (51). Arthroscopic-assisted Broström repair is a type of hybrid technique addressing the intraarticular pathology arthroscopically first and then transitioning to an open Broström repair and combines several advantages of both procedures. Clinical results are excellent, but studies reported a high complication rate (5.3-29.0\%) caused by neurological entrapment and prominent implants (23, 47). The comparative study by Guelfi et al. (51) showed that AOFAS and VAS scores improved significantly in both groups compared to preoperative levels. However, no significant difference in clinical outcomes between the all-arthroscopic and arthroscopic-assisted Broström group was found. Complication rates were significantly smaller in the all-arthroscopic group with $5.3 \%$ vs $40 \%$ in the arthroscopic-assisted group. The only complication in the all-arthroscopic group involved a painful ankle plantarflexion deficit $>10$ degrees. On the contrary, in the arthroscopic-assisted group, complications included plantarflexion deficit, transient neuritis of the superficial peroneal nerve and prominent suture knots requiring anchor removal. None of the patients required ligament revision surgery, and all patients were able to return to activity without limitations. Although the complication rate is significantly higher in the arthroscopic-assisted group in this study, randomized controlled trials are needed to confirm the potential superiority of all-arthroscopic repair.

\section{Limitations}

The present article includes several limitations. First, due to the novelty of all-arthroscopic Broström repair and thus the paucity of studies comparing this method to the more renowned open Broström repair, randomized trials as well as non-randomized cohort studies were included, which might increase the risk for selection bias and confounding. Therefore, MINORS scores were calculated for all non-

\begin{tabular}{|c|c|c|c|c|c|c|}
\hline \multirow[b]{2}{*}{ Study or Subgroup } & \multicolumn{2}{|c|}{ All-inside } & \multicolumn{2}{|c|}{ Open } & \multirow[b]{2}{*}{ Weight } & \multirow{2}{*}{ Odds Ratio } \\
\hline & Events & Total & Events: & Total & & \\
\hline U et al. 2017 & 0 & 23 & 0 & 37 & & Not \\
\hline 2016 & 2 & 19 & 1 & 1B & 11. By & $2.00[0$ \\
\hline Rkgby & 1 & 30 & 2 & 32 & $23.9 x$ & $0.52[0.0$ \\
\hline Woo e & 0 & 2 & 0 & 26 & & No \\
\hline 2020 & 3 & 32 & 2 & 35 & $22.1 \mathrm{x}$ & $1.71[0$. \\
\hline 2016 & 3 & 25 & 2 & 23 & $23.4 x$ & $1.43[0.22$ \\
\hline Zepn & $\mathbf{1}$ & 17 & 0 & 10 & $7.3 \mathbf{x}$ & $1.91[0.07,51$ \\
\hline Zhou et & 1 & 31 & 1 & 36 & $11.5 \%$ & $1.17[0.07,15$ \\
\hline
\end{tabular}

\section{$F=0 x$}

$0.01 \underset{\text { Favours [All-inside] }}{0.1} \stackrel{1}{10} 100$

\section{Figure 7}

Results of aggregate analysis for the comparison of rates for irritation of peroneal nerve and tendons between BR and ST groups. Numbers for 'events' refer to failure; numbers for 'total' refer to total participants. $\mathrm{M}-\mathrm{H}$, Mantel-Haenszel method. 


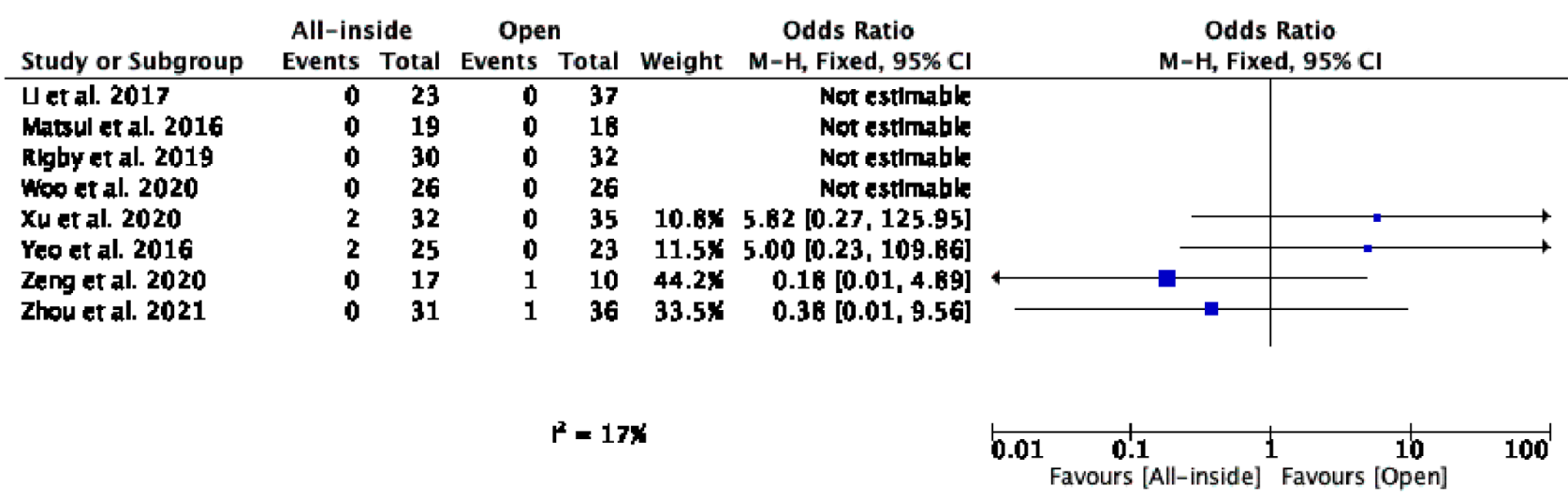

\section{Figure 8}

Results of aggregate analysis for the comparison of knot pain rates between BR and ST groups. Numbers for 'events' refer to failure; numbers for 'total' refer to total participants. $\mathrm{M}-\mathrm{H}$, Mantel-Haenszel method.

randomized trials to assess risk of bias (36). The seven included non-randomized studies have scores ranging from 17 to 22, indicating tolerable scores, although the risk of bias is evidently present. Loss of points was mainly due to the lack of prospective data collection and absence of prospective calculation of study size. In all seven nonrandomized cohort studies, data collection was conducted retrospectively and no adjustment for confounding variables was performed. However, comparison groups were present in all studies.

Secondly, only eight studies were included in total. Moreover, all studies showed high heterogeneity. Altogether, more patients receiving open Broström repair (217 vs 203 in the arthroscopic group) were included in the selected studies. Furthermore, not all mentioned clinical scores were evaluated in all of the studies, which might cause difficulties in drawing valid conclusions. Additionally, a meta-analysis was not performed.

\section{Conclusions}

Similar to open repair, all-arthroscopic ligament repair for CLAl is a safe treatment option that yields excellent clinical outcomes.

\section{ICMJE Conflict of Interest Statement}

R S works as a paid consultant for Arthrex. A L has received Institutional educational grants by Alphamed, Johnson \& Johnson, Medacta, Implantec. All other authors declare no conflicts of interest relevant to this work.

\section{Funding Statement}

This work did not receive any specific grant from any funding agency in the public, commercial or not-for-profit sector.

\section{References}

1. Guillo S, Bauer T, Lee JW, Takao M, Kong SW, Stone JW, Mangone PG, Molloy A, Perera A, Pearce CJ et al. Consensus in chronic ankle instability: aetiology, assessment, surgical indications and place for arthroscopy. Orthopaedics and Traumatology, Surgery and Research 201399 (8 Supplement) S411-S419. (https://doi.org/10.1016/j. otsr.2013.10.009)

2. Ferran NA, Oliva F \& Maffulli N. Ankle instability. Sports Medicine and Arthroscopy Review 200917 139-145. (https://doi.org/10.1097/JSA.0b013e3181a3d790)

3. Karlsson J \& Sancone M. Management of acute ligament injuries of the ankle. Foot and Ankle Clinics 200611 521-530. (https://doi.org/10.1016/j.fcl.2006.07.008)

4. Brostrom L. Sprained ankles. V. Treatment and prognosis in recent ligament ruptures. Acta Chirurgica Scandinavica 1966132 537-550.

5. DiGiovanni BF, Partal G \& Baumhauer JF. Acute ankle injury and chronic lateral instability in the athlete. Clinics in Sports Medicine 200423 1-19, v. (https://doi. org/10.1016/S0278-5919(03)00095-4)

6. Martin RL, Davenport TE, Paulseth S, Wukich DK, Godges JJ \& Orthopaedic Section American Physical Therapy Association. Ankle stability and movement coordination impairments: ankle ligament sprains. Journal of Orthopaedic and Sports Physical Therapy 201343 A1-A40. (https://doi.org/10.2519/jospt.2013.0305)

7. Maffulli N \& Ferran NA. Management of acute and chronic ankle instability. Journal of the American Academy of Orthopaedic Surgeons 200816 608-615. (https://doi. org/10.5435/00124635-200810000-00006)

8. Balduini FC, Vegso JJ, Torg JS \& Torg E. Management and rehabilitation of ligamentous injuries to the ankle. Sports Medicine 19874 364-380. (https://doi. org/10.2165/00007256-198704050-00004)

9. Cox JS. Surgical and nonsurgical treatment of acute ankle sprains. Clinical Orthopaedics and Related Research 1985198 118-126. (https://doi.org/10.1097/00003086198509000-00018)

10. O'Loughlin PF, Murawkski CD, Egan C \& Kennedy JG. Ankle instability in sports. Physician and Sportsmedicine 200937 93-103. (https://doi.org/10.3810/ psm.2009.06.1715)

11. Takao M, Miyamoto W, Matsui K, Sasahara J \& Matsushita T. Functional treatment after surgical repair for acute lateral ligament disruption of the 
ankle in athletes. American Journal of Sports Medicine $2012 \mathbf{4 0}$ 447-451. (https://doi. org/10.1177/0363546511428581)

12. De Vries JS, Krips R, Sierevelt IN, Blankevoort L \& van Dijk CN. Interventions for treating chronic ankle instability. Cochrane Database of Systematic Reviews $2011 \mathbf{8}$ CD004124. (https://doi.org/10.1002/14651858.CD004124.pub3)

13. Gould N, Seligson D \& Gassman J. Early and late repair of lateral ligament of the ankle. Foot and Ankle $1980 \mathbf{1}$ 84-89. (https://doi. org/10.1177/107110078000100206)

14. Cottom JM \& Rigby RB. The'all inside'arthroscopic Broström procedure: a prospective study of 40 consecutive patients. Journal of Foot and Ankle Surgery $2013 \mathbf{5 2} 568-574$. (https://doi.org/10.1053/j.jfas.2013.02.022)

15. Broström L. Sprained ankles. VI. Surgical treatment of'chronic'ligament ruptures. Acta Chirurgica Scandinavica 1966132 551-565.

16. Hamilton WG, Thompson FM \& Snow SW. The modified Brostrom procedure for lateral ankle instability. Foot and Ankle $1993 \mathbf{1 4}$ 1-7. (https://doi. org/10.1177/107110079301400101)

17. Karlsson J, Bergsten T, Lansinger 0 \& Peterson L. Reconstruction of the lateral ligaments of the ankle for chronic lateral instability. Journal of Bone and Joint Surgery: American Volume 198870 581-588. (https://doi.org/10.2106/00004623-19887004000015)

18. Yasui Y, Murawski CD, Wollstein A, Takao M \& Kennedy JG. Operative treatment of lateral ankle instability. JBJS Reviews 20164 1-11. (https://doi.org/10.2106/ JBJS.RVW.15.00074)

19. Hawkins RB. Arthroscopic stapling repair for chronic lateral instability. Clinics in Podiatric Medicine and Surgery 1987 4875-883.

20. Ferkel RD \& Fasulo GJ. Arthroscopic treatment of ankle injuries. Orthopedic Clinics of North America 199425 17-32. (https://doi.org/10.1016/S0030-5898(20)31863-0)

21. Lui TH. Modified arthroscopic Brostrom procedure. Foot and Ankle Surgery 201521 216-219. (https://doi.org/10.1016/j.fas.2015.01.008)

22. Acevedo JI \& Mangone PG. Arthroscopic lateral ankle ligament reconstruction. Techniques in Foot and Ankle Surgery 201110 111-116. (https://doi.org/10.1097/ BTF.0b013e318229bdb8)

23. Corte-Real NM \& Moreira RM. Arthroscopic repair of chronic lateral ankle instability. Foot and Ankle International 200930 213-217. (https://doi.org/10.3113/ FAl.2009.0213)

24. Kashuk KB, Carbonell JA \& Blum JA. Arthroscopic stabilization of the ankle. Clinics in Podiatric Medicine and Surgery 199714 459-478.

25. Vega J, Golanó P, Pellegrino A, Rabat E \& Pena F. All-inside arthroscopic lateral collateral ligament repair for ankle instability with a knotless suture anchor technique. Foot and Ankle International 201334 1701-1709. (https://doi. org/10.1177/1071100713502322)

26. Cottom JM, Baker JS \& Richardson PE. The 'all-inside' arthroscopic Brostrom procedure with additional suture anchor augmentation: a prospective study of 45 consecutive patients. Journal of Foot and Ankle Surgery 201655 1223-1228. (https://doi. org/10.1053/j.jfas.2016.07.023)

27. Cottom JM \& Richardson PE. The 'all-inside' arthroscopic Brostrom procedure augmented with a proximal suture anchor: an innovative technique. Journal of Foot and Ankle Surgery 201756 408-411. (https://doi.org/10.1053/j.jfas.2016.10.013)

28. Lee KT, Kim ES, Kim YH, Ryu JS, Rhyu IJ \& Lee YK. All-inside arthroscopic modified Brostrom operation for chronic ankle instability: a biomechanical study. Knee
Surgery, Sports Traumatology, Arthroscopy 201624 1096-1100. (https://doi.org/10.1007/ s00167-014-3159-2)

29. Cannon LB \& Slater HK. The role of ankle arthroscopy and surgical approach in lateral ankle ligament repair. Foot and Ankle Surgery 200511 1-4. (https://doi.org/10.1016/j. fas.2004.12.001)

30. Hintermann B, Boss AP \& Schäfer D. Arthroscopic findings in patients with chronic ankle instability. American Journal of Sports Medicine $2002 \mathbf{3 0}$ 402-409. (https:// doi.org/10.1177/03635465020300031601)

31. Pitts CC, McKissack HM, Anderson MC, Buddemeyer KM, Bassetty C, Naranje SM \& Shah A. Anatomical structures at risk in the arthroscopic BroströmGould procedure: a cadaver study. Foot and Ankle Surgery 202026 343-346. (https://doi. org/10.1016/j.fas.2019.04.008)

32. Yammine K \& Assi C. Neurovascular and tendon injuries due to ankle arthroscopy portals: a meta-analysis of interventional cadaveric studies. Surgical and Radiologic Anatomy 201840 489-497. (https://doi.org/10.1007/s00276-018-2013-5)

33. De Leeuw PAJ, Golanó P, Sierevelt IN \& van Dijk CN. The course of the superficial peroneal nerve in relation to the ankle position: anatomical study with ankle arthroscopic implications. Knee Surgery, Sports Traumatology, Arthroscopy 201018 612-617. (https://doi.org/10.1007/s00167-010-1099-z)

34. Zengerink M \& van Dijk CN. Complications in ankle arthroscopy. Knee Surgery, Sports Traumatology, Arthroscopy 201220 1420-1431. (https://doi.org/10.1007/s00167012-2063-x)

35. Moher D, Shamseer L, Clarke M, Ghersi D, Liberati A, Petticrew M, Shekelle P, Stewart LA \& PRISMA-P Group. Preferred reporting items for systematic review and meta-analysis protocols (PRISMA-P) 2015 statement. Systems Research 20154 1. (https://doi.org/10.1186/2046-4053-4-1)

36. Slim K, Nini E, Forestier D, Kwiatkowski F, Panis Y \& Chipponi J. Methodological index for non-randomized studies (minors): development and validation of a new instrument. ANZ Journal of Surgery 200373 712-716. (https://doi.org/10.1046/ j.1445-2197.2003.02748.x)

37. Zhou YF, Zhang ZZ, Zhang HZ, Li WP, Shen HY \& Song B. All-inside arthroscopic modified Broström technique to repair anterior talofibular ligament provides a similar outcome compared with open Broström-Gould procedure. Arthroscopy $202137268-279$. (https://doi.org/10.1016/j.arthro.2020.08.030)

38. Woo BJ, Lai MC \& Koo K. Arthroscopic versus open Broström-Gould repair for chronic ankle instability. Foot and Ankle International 202041 647-653. (https://doi. org/10.1177/1071100720914860)

39. Zeng G, Hu X, Liu W, Qiu X, Yang T, Li C \& Song W. Open Broström-Gould repair vs arthroscopic anatomical repair of the anterior talofibular ligament for chronic lateral ankle instability. Foot and Ankle International 202041 44-49. (https://doi. org/10.1177/1071100719875964)

40. Xu C, Li M, Wang C \& Liu H. A comparison between arthroscopic and open surgery for treatment outcomes of chronic lateral ankle instability accompanied by osteochondral lesions of the talus. Journal of Orthopaedic Surgery and Research 202015 113. (https://doi. org/10.1186/s13018-020-01628-3)

41. Rigby RB \& Cottom JM. A comparison of the 'all-inside' arthroscopic Broström procedure with the traditional open modified Broström-Gould technique: a review of 62 patients. Foot and Ankle Surgery 201925 31-36. (https://doi.org/10.1016/j. fas.2017.07.642) 
42. Li H, Hua Y, Li H, Ma K, Li S \& Chen S. Activity level and function 2 years after anterior talofibular ligament repair: a comparison between arthroscopic repair and open repair procedures. American Journal of Sports Medicine 201745 2044-2051. (https://doi. org/10.1177/0363546517698675)

43. Yeo ED, Lee KT, Sung IH, Lee SG \& Lee YK. Comparison of all-inside arthroscopic and open techniques for the modified Broström procedure for ankle instability. Foot and Ankle International 201637 1037-1045. (https://doi. org/10.1177/1071100716666508)

44. Matsui K, Takao M, Miyamoto W \& Matsushita T. Early recovery after arthroscopic repair compared to open repair of the anterior talofibular ligament for lateral instability of the ankle. Archives of Orthopaedic and Trauma Surgery 2016136 93-100. (https://doi.org/10.1007/s00402-015-2342-3)

45. Hua Y, Chen S, Li Y, Chen J \& Li H. Combination of modified Broström procedure with ankle arthroscopy for chronic ankle instability accompanied by intraarticular symptoms. Arthroscopy 201026 524-528. (https://doi.org/10.1016/j. arthro.2010.02.002)

46. Giza E, Shin EC, Wong SE, Acevedo Jl, Mangone PG, Olson K \& Anderson MJ. Arthroscopic suture anchor repair of the lateral ligament ankle complex: a cadaveric study. American Journal of Sports Medicine 201341 2567-2572. (https://doi. org/10.1177/0363546513500639)

47. Kim ES, Lee KT, Parks JS \& Lee YK. Arthroscopic anterior talofibular ligament repair for chronic ankle instability with a suture anchor technique. Orthopedics 2011 34 1-9. (https://doi.org/10.3928/01477447-20110228-03)

48. Santos FF \& Santos NR. Arthroscopic treatment of lateral ankle instability. Is there a safe zone? An anatomic study. Foot and Ankle Surgery 202026 61-65. (https://doi. org/10.1016/j.fas.2018.11.011)

49. Acevedo Jl, Ortiz C, Golano P \& Nery C. ArthroBroström lateral ankle stabilization technique: an anatomic study. American Journal of Sports Medicine 201543 2564-2571. (https://doi.org/10.1177/0363546515597464)

50. Drakos M, Behrens SB, Mulcahey MK, Paller D, Hoffman E \& DiGiovanni CW. Proximity of arthroscopic ankle stabilization procedures to surrounding structures: an anatomic study. Arthroscopy 201329 1089-1094. (https://doi.org/10.1016/j.arthro.2013.02.011)

51. Guelfi M, Nunes GA, Malagelada F, Cordier G, Dalmau-Pastor $M$ \& Vega J. Arthroscopic-assisted versus all-arthroscopic ankle stabilization technique. Foot and Ankle International 202041 1360-1367. (https://doi. org/10.1177/1071100720938672) 